\title{
Transient pancytopenia preceding adult acute lymphoblastic leukemia with chromosomal abnormalities including the Philadelphia chromosome: A case report and review of the literature
}

\author{
YUN LIANG, LUYIN DING, XIAN LI, WEIQIN WANG and XIAOHONG ZHANG \\ Department of Hematology, The Second Affiliated Hospital of Zhejiang University School of Medicine, \\ Hangzhou, Zhejiang 310009, P.R. China
}

Received November 11, 2014; Accepted September 9, 2015

DOI: $10.3892 / 01.2015 .3766$

\begin{abstract}
A preleukaemic phase, typified by transient pancytopenia, is a rare occurrence that usually affects children and adolescents. The present study reports the case of a 50-yearold woman with transient pancytopenia, which manifested as a fever, cough and severe anemia. Three weeks following treatment of pancytopenia with antibiotics, red blood cell and platelet transfusion, granulocyte colony-stimulating factor and human $\gamma$ globulin, the condition of the patient was improved. However, 3 weeks following discharge from hospital, the patient was diagnosed with acute lymphoblastic leukemia (ALL) with complex chromosomal abnormalities, including Philadelphia chromosome and P190 breakpoint cluster region-ABL. Complete remission was achieved following one course of combination chemotherapy. In conclusion, adult ALL with pancytopenia as a preceding symptom is rare, difficult to diagnose early and easily misdiagnosed. In addition, the pathogenesis of ALL and the precipitating factors underlying this disease require further investigation.
\end{abstract}

\section{Introduction}

It is understood that myelodysplastic syndrome (MDS) may be considered to precede acute non-lymphoblastic leukemia (pre-ANLL), and this concept has reached a consensus $(1,2)$. However, to date, pre-ALL remains a rare and uncertain condition. A number of studies have demonstrated that transient pancytopenia, another bone marrow disorder, may be associated with ALL in children and adolescents $(3,4)$. The typical pancytopenic phase, which lasts from several weeks to several

Correspondence to: Dr Yun Liang, Department of Hematology, The Second Affiliated Hospital of Zhejiang University School of Medcine, 88 Jiefang Road, Hangzhou, Zhejiang 310009, P.R. China E-mail: liangyun@zju.edu.cn

Key words: pancytopenia, Philadelphia chromosome, acute lymphoblastic leukemia months, is transient, followed by spontaneous remission or remission induced by corticosteroids, and subsequent transformation into common ALL (5). The prognosis of patients with ALL with or without pre-ALL is similar following standard chemotherapy (5). The situation can not be determined in adult patients because such cases are exceedingly rare.

Adult ALL with pancytopenia as a preceding symptom is rare, difficult to diagnose early and prone to misdiagnosis (6-8). The present study reports a case of transient pancytopenia preceding adult ALL with complex chromosomal abnormalities, including Philadelphia chromosome and P190 breakpoint cluster region (BCR)-ABL. The genetic characteristics and clinical outcome of the patient are summarized. The present study aimed to improve understanding of the etiology and pathogenesis underlying adult ALL.

\section{Case report}

A 50-year-old female was admitted The Second Affiliated Hospital of Zhejiang University School of Medicine (Hangzhou, China) on 27 Februrary 2013, with a cough, fever, fatigue and severe anemia for 1 week. The patient experienced chills and a fever up to $39.5^{\circ} \mathrm{C}$ with cough, sputum, chest tightness and shortness of breath, which were resistant to antibiotics (ceftazidine administered via injection, 1.0 g every $8 \mathrm{~h}$ ). Physical examination revealed an anemic complexion, slight petechiae in the skin and moist rales in the lungs. No enlargement of the lymph nodes, liver or spleen was detected. No abnormalities were identified in the patient's medical history or family history. Laboratory tests were performed on the day of admittance and revealed anemia with reticulocytopenia (hemoglobin, $30 \mathrm{~g} / \mathrm{l}$; normal range, 110-160 g/l), leucopenia (white blood cells, $0.4 \times 10^{9} / 1$; normal range, $\left.4.0-10.0 \times 10^{9} / 1\right)$ and thrombocytopenia (platelets, $17 \times 10^{9} / 1$; normal range, $100-300 \times 10^{9} / 1$ ). The results of blood biochemical analysis indicated hypoalbuminemia (albumin, $26.6 \mathrm{~g} / \mathrm{l}$; normal range, 35.0-52.0 g/l) and a marked increase in C-reactive protein levels $(122.5 \mathrm{mg} / \mathrm{l}$; normal range, $<6.0 \mathrm{mg} / \mathrm{l})$. An initial bone marrow (BM) smear and biopsy showed pancytopenia, significantly reduced numbers of nucleated cells, significantly decreased precursor myelocytes and relatively increased lymphocyte levels, while no marked 
Table I. Complete blood counts of the present patient at the time of initial diagnosis of AAH, at discharge following recovery of pancytopenia and at the established diagnosis of ALL.

\begin{tabular}{lccc}
\hline Parameter & At diagnosis of AAH & Following recovery & At diagnosis of ALL \\
\hline Time, weeks & 0 & 3 & 7 \\
Hemoglobin, $\mathrm{g} / \mathrm{l}$ & 30 & 98 & 104 \\
Leukocytes, $\mathrm{x} 10^{9} / 1$ & 0.4 & 5.6 & 291.3 \\
Neutrophils, $\mathrm{x} 10^{9} / 1$ & & 4.9 & 24.7 \\
Platelets, $\mathrm{x} 10^{9} / 1$ & 17 & 128 & 18 \\
Peripheral blood blasts, $\%$ & 0 & 0 & 85 \\
\hline
\end{tabular}

AAH, acute arrest of hemopoiesis; ALL, acute lymphoblastic leukemia.

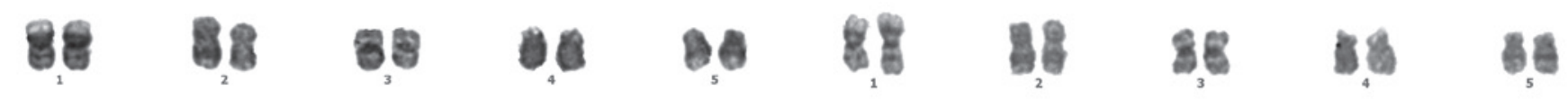

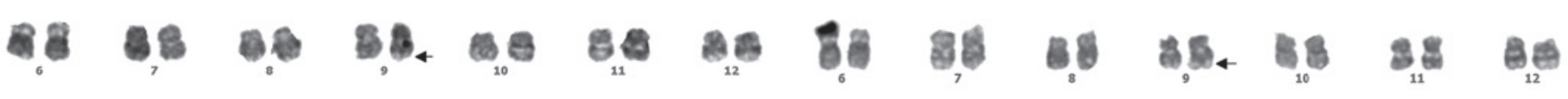 8
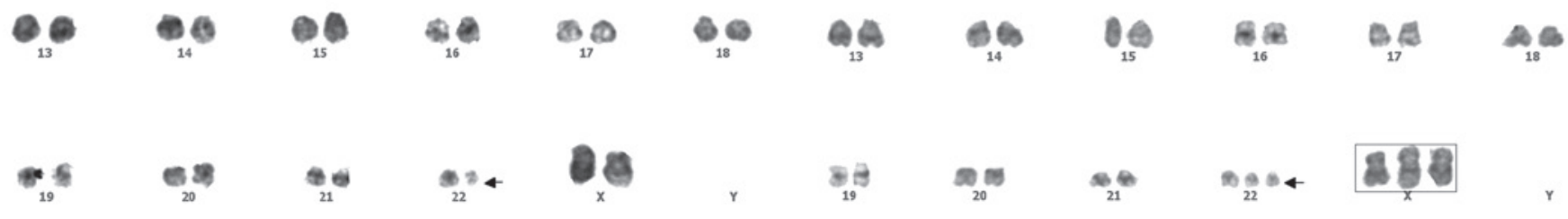

Figure 1. Karyotype analysis revealed 46, XX, t(9;22) (q34;q11.2) [6]/48, idem,+X,+der (22) t(9;22) [10]/46, XX [4]. Arrows indicate Philadelphia chromosome.

abnormalities were identified in erythroid or megakaryocytic hematopoietic cell distribution. Flow cytometric and karyotype analyses were not able to be conducted due to the scarcity of nucleated cells. Cluster of differentiation 55 (CD55)/CD59 expression in erythrocytes and granulocytes was normal. The urinary hemosiderin test, as well as direct and indirect Coombs' tests were negative. Viral serologic studies, including hepatitis $\mathrm{A}, \mathrm{B}$ and $\mathrm{C}$ virus, human immunodeficiency virus, Epstein-Barr virus and cytomegalovirus were negative, and fluorescent nucleic acid detection of parvovirus B19 was also negative. A pulmonary computed tomography (CT) scan revealed infectious lesions in the lungs, with little pleural effusion. B-mode ultrasound showed no notable abnormalities of the liver, gallbladder, pancreas or spleen and no significant enlargements of the lymph nodes. Acute arrest of hemopoiesis (AAH) and pulmonary infection were diagnosed. The patient was therefore treated with antibiotics (imipenem and cilastatin sodium administered via injection, $0.5 \mathrm{~g}$ every $8 \mathrm{~h}$ ), red blood cell and platelet transfusion, granulocyte colony-stimulating factor and human $\gamma$ globulin. The condition of the patient rapidly improved, and white blood cell, hemoglobin and platelet levels had increased three weeks later (Table I). The BM was reviewed and revealed normal hematopoiesis without excessive blast cells or dysplasia. Re-examination via pulmonary CT indicated that the infectious lesions had significantly remitted. The patient was subsequently discharged 22 days following discharge and normal peripheral blood test results were achieved at weekly follow-ups.
However the patient was readmitted high fever three weeks following discharge. Routine blood tests revealed leukocytosis, mild anemia and thrombocytopenia. The peripheral blood test identified a white blood cell count of $291.3 \times 10^{9} / 1$ (normal range, 4.0-10.0 $\times 10^{9} / 1$ ), hemoglobin levels of $104 \mathrm{~g} / \mathrm{l}$ (normal range, 110-160 g/l), platelet levels of $18 \times 10^{9} / 1$ (normal range, $100-300 \times 10^{9} / 1$ ) and $85 \%$ blast cells (Table I, day 50). Extensive lymphadenopathy and splenomegaly were confirmed by B-mode ultrasound. BM examination showed extreme hyperplasia with $65 \%$ excess blast cells and inhibited normal hematopoiesis function. Blast cells accounted for $87 \%$ of bone marrow cells, as indicated by immunophenotyping, which had positive expression of membrane antigens, including HLA-DR, CD10, CD15, CD19, CD20, CD22, CD34, CD38, CD123, CD79a and terminal deoxynucleotidyl transferase. All available leukemia fusion genes were examined, and positive BCR/ABL (P190) was detected. The remaining fusion genes, including MLL/AFX, MLL/AF1P, MLL/AF4, dupMLL, MLL/ENL, E2A/PBX1, SIL/TAL1, HOX11, TEL/AML1 and TEL/ABL, were all negative. Complex chromosomal mutations were detected during karyotype analysis (Fig. 1). A final diagnosis of acute B-cell lymphoblastic leukemia was reached. The patient refused imatinib treatment due to economic problems, and was therefore administered VDP regimen chemotherapy $(50 \mathrm{mg}$ daunorubicin on days 1-3; $2 \mathrm{mg}$ vincristin on days $1,8,15$ and 22; $60 \mathrm{mg}$ prednisone on days $1-21$ and $30 \mathrm{mg}$ prednisone 
on days 22-28). Although complete remission was achieved following one therapeutic course, the patient discontinued treatment and was lost to follow-up.

\section{Discussion}

A number of studies have indicated that certain cases of ALL may manifest preceding symptoms of hematopoietic disorders (pre-ALL), and that the prevalence of pre-ALL is $1.3-2.2 \%$ among children with ALL (3). Pre-ALL is characterized by fever with pancytopenia as the primary symptom, which is typically observed in children $<10$ years old, and has markedly higher incidence amongst girls. ALL with pre-ALL has a similar prognosis compared with that of ALL patients without preceding symptoms of pancytopenia (5).

In the present case, laboratory tests identified neutropenia, severe anemia and a relatively mild decrease or no decrease at all in platelets. BM smear and biopsy showed significantly reduced numbers of nucleated cells, but no notable morphological abnormalities. A previous study reported that the majority of these patients restored normal hematopoiesis within 1 month following treatment, but subsequently developed ALL within 6 months (3-5).

However, cases of adult ALL manifesting pancytopenia are rare, and may be easily neglected or misdiagnosed; few cases were available in the literature $(8,9)$. In the present case, symptoms of leukemia were not identified in peripheral blood or BM on initial examination. The patient presented with pancytopenia in peripheral blood and suppressed myeloproliferation in the BM, but these symptoms were rapidly restored to normal following active treatment. The initial diagnosis was, therefore, AAH. AAH is also termed spontaneous remission of aplastic anemia, the attacks last several weeks and may be associated with external factors such as drug or infection (10). Certain risk factors may result in hemopoiesis dysfunction or decompensation, which manifests as a transient decrease or absence of hematopoietic cells. This situation may attenuate once the factors have been removed (11). Common risk factors include infection (particularly viruses), drugs, various nutrients deficiencies and immune disorders (11). In the present case, three weeks following admission, normal hematopoiesis in the BM was restored. However, once the disease progressed, it was able to be diagnosed as acute leukemia, characterized by the abnormal proliferation of leukocytes.

In addition, complex chromosomal abnormalities, including the Philadelphia $(\mathrm{Ph})$ chromosome, were identified by karyotype analysis in the present case. Therefore, when a patient is diagnosed with AAH without clear etiology, the disease should be monitored more carefully and more detailed BM examinations, including immunophenotyping and karyotype analysis, should be conducted. Ph chromosome is characteristic of chronic myeloid leukemia, which may also be observed in 2-5\% of ALL in children and 15-30\% of adult ALL (12). Positivity for the Philadelphia chromosome is an independent prognostic indicator for adult ALL, predicting the poor efficacy of conventional chemotherapy, low remission rate and decreased time prior to relapse. Imatinib-based combined chemotherapy followed by allogeneic hematopoietic stem cell transplantation is the first-line treatment for Philadelphia chromosome-positive ALL (13).
Associations between preceding symptoms of hematopoietic disorders and subsequent ALL remain controversial. Whether the pre-ALL period and the subsequent ALL period are distinct clinical manifestations of one disease or not requires further clarification. Since it is difficult to explain how pre-ALL transforms into ALL over the period of a few days to several weeks, the hypothesis that leukemia occurs prior to pre-ALL may be plausible (14). Numerous retrospective studies in children have indicated that pediatric patients have normal chromosomes during the pancytopenic period and then abnormal karyotypes may be detected in the ALL period $(3,14)$. For this reason, it has been suggested that the former and the latter are distinct stages of one disease. For example, cells with a normal karyotype proliferate in the forms of colonization, followed by abnormal chromosome changes that manifest as the clinical symptoms of typical ALL $(14,15)$. It has therefore been hypothesized that the pancytopenic period observed in such patients occurred as a result of the inherent characteristics of leukemia cells.

Certain studies have reported that parvovirus B19 infection is associated with the inhibited status of bone marrow in pre-ALL children (16), and that children infected by parvovirus B19 are more susceptible to severe anemia (17). However, there was no evidence of such viral infection in the present patient.

In conclusion, to the best of our knowledge, this was the first report of adult transient pancytopenia preceding ALL with complicated chromosomal abnormalities, including the Philadelphia chromosome and P190 BCR-ABL. Further investigations are required to elucidate the etiology and pathology of pre-ALL in adults.

\section{References}

1. Wegelius R: Bone marrow dysfunctions preceding acute leukemia in children: A clinical study. Leuk Res 16: 71-76, 1992.

2. Shi J, Shao ZH, Liu H, Bai J, Cao YR, He GS, Tu MF, Wang L, Hao YS, Yang TY and Yang CL: Transformation of myelodysplastic syndromes into acute myeloid leukemias. Chin Med J (Engl) 117: 963-967, 2004.

3. Hasle H, Heim S, Schroeder H, Schmiegelow K, Ostergaard E and Kerndrup G: Transient pancytopenia preceding acute lymphoblastic leukemia (pre-ALL). Leukemia 9: 605-608, 1995.

4. Villarreal-Martínez L, Jaime-Pérez JC, Rodríguez-Martínez M, González-Llano O and Gómez-Almaguer D: Acute lymphoblastic leukemia of childhood presenting as aplastic anemia: Report of two cases. Rev Bras Hematol Hemoter 34: 165-167, 2012.

5. Armata J, Grześkowiak-Melanowska J, Balwierz W, Najbar-Pabian A and Pawlik-Niesytto E: Prognosis in acute lymphoblastic leukemia (ALL) in children preceded by an aplastic phase. Leuk Lymphoma 13: 517-518, 1994.

6. Zhao H, Zhang L, Zhu X, Chen Y, Zou Y, Zhang L, Yang R and Han ZC: Transient pancytopenia preceding $T$ lineage acute lymphoblastic leukemia. Acta Haematol 112: 167-169, 2004.

7. Li Q, Chen Z, You Y and Zou P: Transient pancytopenia preceding acute lymphoblastic leukemia with positive Philadelphia chromosome. Leuk Res 32: 1317-1320, 2008.

8. Kelly K and Murphy P: Aplastic anaemia preceding acute lymphoblastic leukaemia in an adult with isolated deletion of chromosome 9q. Leuk Res 32: 1936-1938, 2008.

9. Sohn SK, Suh JS, Lee J and Lee KB: Pancytopenic prodrome (pre-ALL) of acute lymphoblastic leukemia in adults: possible pathogenesis. Korean J Intern Med 13: 64-67,1998.

10. Lee JH, Lee JH, Shin YR, Lee JS, Kim WK, Chi HS, Park CJ and Lee KH: Spontaneous remission of aplastic anemia: A retrospective analysis. Haematologica 86: 928-933, 2001. 
11. Bozkaya H, Yurdaydin C, Törüner M, Arat M, Bozdayi AM Erekul S, Cinar K, Koç H and Uzunalimoğlu O: Remission of severe aplastic anemia associated with hepatitis B virus infection after viral clearance: Potential role of lamivudine. Dig Dis Sci 47: 1782-1785, 2002.

12. Majlis A, Smith TL, Talpaz M, O'Brien S, Rios MB and Kantarjian HM: Significance of cytogenetic clonal evolution in chronic myelogenous leukemia. J Clin Oncol 14: 196-203, 1996.

13. Adele K: Current treatment of Philadelphia chromosome-positive acute lymphoblastic leukemia. Haematologica 95: 8-12, 2010

14. Matloub YH, Brunning RD, Arthur DC and Ramsay NK: Severe aplastic anemia preceding acute lymphoblastic leukemia. Cancer 71: 264-268, 1993.
15. Horsley SW, Colman S, McKinley M, Bateman CM, Jenney M, Chaplin T, Young BD, Greaves M and Kearney L: Genetic lesions in a preleukemic aplasia phase in a child with acute lymphoblastic leukemia. Genes Chromosomes Cancer 47: 333-40, 2008.

16. Heegaard ED, Madsen HO and Schmiegelow K: Transient pancytopenia preceding acute lymphoblastic leukaemia (pre-ALL) precipitated by parvovirus B19. Br J Haematol 114: 810-813, 2001.

17. Kishore J, Sen M, Kumar A and Kumar A: A pilot study on parvovirus B19 infection in paediatric haematological malignancies. Indian J Med Res 133: 407-413, 2011. 